\title{
Silencing approach using Poly (ADP-ribose) polymerase gene to improve drought stress tolerance in maize
}

\author{
Sylvester Elikana Anami \\ Supervisor: Dirk Inzé
}

(I) Department of Plant Biotechnology and Genetics, Ghent University

Public defence: 23 April 2010

Drought is one of the environmental stresses that have a profound negative effect on the growth and development of plants, and, in most regions of the world, the productivity of major crops is defined by their ability to cope with this stress. However, commercially improved crop performance under drought condition has been challenging because of the low heritability of the trait, the unpredictable nature of most periods of drought stress encountered in growing areas and gaps in our understanding of drought biology. As a consequence, a number of approaches have been tested for improving the performance of crops grown under periodic drought conditions.

Chapter I of this $\mathrm{PhD}$ thesis reviews abiotic constraints that affect growth, development and production of tropical maize and focuses on the mechanisms that regulate drought stress tolerance in maize. In addition, biotechnological approaches to manage abiotic stress tolerance in tropical maize have been discussed. Though the focus was on tropical maize, the strategies discussed are applicable to temperate maize and other crops of economic importance. Furthermore, due to the complexity of the drought trait and the multitude of factors that influence yield, plant molecular breeding that encompasses the integration of traditional conventional breeding and modern gene technology to improve drought stress tolerance in maize has been proposed in this chapter. The current status of tropical maize transformation using Agrobacterium as a vehicle for DNA transfer is emphasized and finally, the chapter addresses the present status of genetically modified organisms (GMOs) regulation in sub-Saharan Africa. Indeed, a combination of previously reported as well as novel approaches will be needed to increase plant abiotic stress resistance to levels high enough for field application in crops. Such novel technologies include the manipulation of artificial miRNAs (amiRNAs) guided gene regulation to improve plant stress tolerance. AmiRNAs represents a novel, feasible and highly specific approach for effective post-transcriptional gene silencing (PTGS) in plants. This 
approach can effectively modulate agronomically important traits in varieties used in modern breeding programs. In addition, the approach is suited for candidate gene validation, comparative functional genomics between different varieties, and for improvement of agronomic performance. Both transcriptomics and metabolomics are important emerging technologies to understand the physiology of drought stress in plants, especially when performed in time course experiments (Anami et al., 2009).

Transgenic approaches currently offer new opportunities to improve tolerance to abiotic stresses. Therefore, engineering crop plants for high $\mathrm{NAD}^{+}$regeneration, for example, by an efficient upregulation of the $\mathrm{NAD}^{+}$salvage pathway or by a reduced $\mathrm{NAD}+$ consumption under stress conditions, is a valuable approach to enhance overall stress tolerance in crops. Chapter 2 of this thesis therefore aimed to review PARP protein family in plants, their metabolism and their role in abiotic stress tolerance. Indeed, Poly(ADPribosyl)ation (PAR) is a unique posttranslational protein modification mediated by the PARP enzyme that tags long-branched poly(ADP-ribose) polymers to nuclear target proteins.

Gene silencing capability of RNA interference (RNAi) and artificial miRNA are being used to study individual gene's biological function and role in biochemical pathways. The application of gene silencing technology has a positive impact on the improvement of plant productivity or nutritional value. In this study, both RNAi and amiRNA technology have been used to silence maize PARP1 gene. Chapter 3 aimed to introduce and discuss the mechanisms of gene silencing in plants by both RNAi and amiRNA strategies. Bioinformatics and experimental approaches to identify miRNAs and miRNA targets in plants are further addressed in this chapter.

Chapter 4 presents results of optimized in vitro culture conditions for efficient somatic embryogenesis and plant regeneration from tropical maize genotypes (Anami et al., 2008, 2010). The availability of an in vitro regeneration system for tropical maize is a prerequisite for effective genetic transformation. The regeneration of a recalcitrant tropical maize genotype (TL26) was improved by a single cross with inbred lines (CML2I6 and CML244) as maternal donor that regenerate efficiently. The chapter establishes that callus induction from immature embryo of tropical maize genotypes studied are induced from the embryo axis side. A cocultivation of immature embryos of tropical maize genotypes with Agrobacterium strains containing a Gateway vector and a GUS reporter gene with or without intron in its coding region were used to obtain information on the competence of the tissue for infection. Indeed, tropical maize genotypes studied were found to be incompetent to Agrobacterium infection and that particle bombardment need to be integrated in future establishment of genetic transformation of these genotypes. The results presented in this chapter will assist in the achievement of genetic transformation in tropical maize that will accelerate germplasm development and fundamental research.

The study of biological systems relies to a large extent on DNA cloning technologies enabling the analysis of recombinant genes through transgenic research. Chapter 5 summarises the design and cloning of two hpRNAi constructs targeting ZmPARP1 gene within the region that seemed specific at the 5' end of the gene using the Gateway platform. As 
an alternative approach to RNAi technology, the chapter describes the design and cloning of three amiRNAs targeting the ZmPARP1 gene: two designed before the Weigel tool for the design of amiRNA became available for crops, and one amiRNA designed later with the Weigel online tool on the maize genome against the PARP1 alpha-helical domain. As a complementary approach to RNAi and amiRNA technonology, chapter 6 describes the verification of the $\mathrm{Mu}$ insertion site at the 5'position of the maize PARP1 gene that corresponds to disruption of maize PARP1 gene.

Maize may be transformed very efficiently using Agrobacterium tumefaciens-mediated methods despite the fact that it is not a natural host to Agrobacterium tumefaciens. Chapter 7 describes the establishment of improved, efficient and reproducible Agrobacterium tumefaciens-mediated transformation method using maize inbred Bro4 immature embryo's and newly developed GATEWAY ${ }^{\mathrm{TM}}$ vectors with a $\mathrm{p} 35 \mathrm{~S}$-bar selectable marker and the $\mathrm{pU}$ BIL promoter to drive overexpression or hairpin constructs. Stable T-DNA integration in the maize genome was demonstrated by PAT assay and polymerase chain reaction of To shoots and TI progenies. Mendelian TI segregation ratio's were obtained for most transgenic events and showed that most transgenic shoots were clonal and originated from somatic embryogenesis. Predominantly single T-DNA loci were present in the different transgenic events, only a minor fraction, $7 \%$, had a distorted segregation ratio indicating a non-clonal origin through organogenesis. Dried, transformed Tr seeds were obtained 7 months after co-cultivation. A restricted tissue-culture period of 4 months in the procedure prevented somaclonal variation in the To transformed shoots - no defects in morphology or fertility were observed.

Poly(ADP-ribosyl)ation is an important part of the plant response to abiotic and biotic stresses (De Block et al., 2005; Vanderauwera et al., 2007). Indeed silencing of Arabidopsis APP gene leads to plants displaying increased resistance to drought, high light, and oxidative stresses (De Block et al., 2005). The outperforming stress tolerance was due to a maintained energy homeostasis as a result of reduced $\mathrm{NAD}^{+}$consumption and alterations in abscisic acid levels that facilitate the induction of a wide set of defenserelated genes including increased levels of the cyclic nucleotide ADP-ribose (cADPR) (De Block et al., 2005; Vanderauwera et al., 2007). The results described in chapter 8 aimed to assess similar approach in maize through silencing Zea mays PARP1 gene. Performance of transgenic plants under drought and oxidative stress was evaluated and the finding demonstrate that plants transformed with amiRNAI and 3 gene constructs target resulted in downregulation of the PARP1 gene expression and resulted in a few lines with putative improved tolerance to drought and oxidative stress based on plant height and fresh weight parameters.

\section{References}

Anami, E. S., Mguu, J. A., Hanley-Bowdoin, L., Rasha, A. O., Nelissen, H., Inzé, D., Van Lijsebettens, M., \& Machuka, J. (2008). Progress in transformation and regenereation of tropical inbred maize lines in Kenya. Maize Genet. Coop. Newslett. 82: 22-23.

Anami, S., De Block, M., Machuka, J. \& Van Lijsebettens, M. (2009). Molecular improvement of tropical maize for drought stress tolerance in sub-Saharan Africa. Crit. Rev. Plant Sci. 28: 16-35. 
Anami, S., Mgutu, AJ., Taracha, C., Coussens, G., Karimi, M., Hilson, P., Van Lijsebettens, M. \& Machuka, J. (2010). Somatic embryogenesis and plant regeneration of tropical maize genotypes. Plant Cell Tiss Organ Cult 102: 285-295.

De Block, M., Verduyn, C., De Brouwer, D., \& Cornelissen, M. (2005). Poly(ADP-ribose) polymerase in plants affects energy homeostasis, cell death and stress tolerance. Plant J. 41: 95-106.

Vanderauwera, S., De Block, M., Van de Steene, N., van de Cotte, B., Metzlaff, M., \& Van Breusegem, F. (2007). Silencing of poly (ADP-ribose) polymerase in plants alters abiotic stress signal transduction. Proc. Natl. Acad. Sci. USA 104: 15150-15155 [PMID 17823244]. 